\title{
Application of Laplace transforms for the solution of transient mass- and heat-transfer problems in flow systems
}

\author{
SPAS D. KOLEV $\dagger$ and WILLEM E. VAN DER LINDEN \\ Laboratory for Chemical Analysis, Department of Chemical Technology, \\ University of Twente, P.O. Box 217, NL-7500 AE Enschede, The Netherlands
}

(Received 11 February 1991)

\begin{abstract}
A fast numerical techrique for the solution of partial differential equations describing timedependent two- or three-dimensional transport phenomena is developed. It is based on transforming the original time-domain equations into the Laplace domain where numerical integration is performed and by subsequent numerical inverse transformation the final solution can be obtained. The computation time is thus reduced by more than one order of magnitude in comparison with the conventional finite-difference techniques. The effectiveness of the proposed technique is demonstrated by illustrative examples.
\end{abstract}

\section{INTRODUCTION}

THE APPLICATION of the Laplace transforms [1] is one of the classical approaches for the solution of various chemical engineering problems described by linear ordinary or partial differential equations. The Laplace transform, $f(p)$, of a given piece-wise continuous time domain function, $f(\theta)$, which is of exponential order is defined by

$$
\bar{f}(p)=\int_{0}^{\infty} f(\theta) \exp [-p \theta] \mathrm{d} \theta
$$

However, this approach is not universally applicable. Its natural limitations are determined by the possibilities for solving the relevant equations in the Laplace domain and the subsequent performance of the inverse transformation of this solution. Although, in many chemical engineering problems described by ordinary or partial differential equations with constant coefficients it does not pose substantial difficulties to obtain the corresponding Laplace domain solution, these solutions are rather complicated functions of $p$ and their analytical inverse transformation is exceedingly difficult if not impossible. This obstacle could be overcome by applying numerical techniques for the inversion of Laplace transforms [2]. They are usually based on one of the following approaches: expansion of the Laplace transform into series of functions (e.g. orthogonal polynomials); reducing the problem to inverse Fourier transformation for which efficient computational techniques exist ; or numerical solution of the Melline integral

† Permanent address : Faculty of Chemistry, University of Sofia, 1 James Bouchier Ave., BG-1126 Sofia, Bulgaria.

$$
f(\theta)=\int_{\sigma-i x}^{\sigma+i x} \vec{f}(p) \exp [p 0] \mathrm{d} p
$$

where $\sigma$ is the real part of $p$.

It has been shown that for the solution of masstransfer problems in flow, when a flat velocity profile is assumed, the numerical techniques employing expansion of the Laplace domain solution into scrics of Chebyshov polynomials of the first kind or Fourier sine series give best results with respect to precision and consumption of computation time [2].

However, in the solution of linear partial differential equations describing two- or three-dimensional problems, and with coefficients which are not necessarily constants, the Laplace transformation does not lead to readily solvable partial or ordinary differential equations. For this reason in such cases other numerical techniques for approximation of the solution of these partial differential equations are utilized. Among them the finite-difference techniques [3] have gained a considerable popularity. One of the main drawbacks of these techniques is that they require a considerable amount of computation time especially in the cases of multidimensional transient problems. This could be a serious disadvantage if the corresponding equation must be solved many times (e.g. parameter identification).

As partial differential equations of the aforesaid type are frequently encountered in chemical engineering, the necessity of introducing a more efficient numerical approach for their solution is obvious.

In this paper, a fast numerical technique for the solution of linear partial differential equations, combining the Laplace transform and the finite-difference techniques, is proposed. It is illustrated on equations describing the transient mass-transfer in single and multi-stream laminar parallel-plate flow systems. 


\begin{tabular}{|c|c|c|c|}
\hline \multicolumn{4}{|c|}{ NOMENCLATURE } \\
\hline$a$ & half of the distance between the & $t$ & time $[s]$ \\
\hline & parallel plates $[\mathrm{m}]$ & $u$ & average linear flow rate $\left[\mathrm{m} \mathrm{s}^{-1}\right]$ \\
\hline$b_{k}$ & coefficients defined in equation (6) & $U$ & dimensionless flow rate, $1.5\left(1-Y^{2}\right)$ \\
\hline$c$ & solute concentration $\left[\mathrm{mol} \mathrm{m}^{-3}\right]$ & $x$ & axial coordinate $[\mathrm{m}]$ \\
\hline$c_{0}$ & initial solute concentration $\left[\mathrm{mol} \mathrm{m}^{-3}\right]$ & $X$ & dimensionless axial coordinate, $x / L$ \\
\hline C & dimensionless solute concentration, $c / c_{0}$ & $y$ & coordinate perpendicular to the parallel \\
\hline $\bar{C}$ & Laplace transform of $C$ & & plates $[\mathrm{m}]$ \\
\hline$D_{\mathrm{m}}$ & molecular diffusion coefficient $\left[\mathrm{m}^{2} \mathrm{~s}^{-1}\right]$ & $Y$ & dimensionless coordinate perpendicular \\
\hline$f(\theta)$ & time-domain function & & to the parallel plates, $y / a$. \\
\hline $\bar{f}(p)$ & Laplace transform of $f(\theta)$ & & \\
\hline$K$ & number of time-steps & \multicolumn{2}{|c|}{ Greek symbols } \\
\hline$L$ & characteristic length $[\mathrm{m}]$ & $\beta$ & geometrical dimensionless group, $(a / L)^{2}$ \\
\hline$M$ & number of $x$-steps & $\theta$ & dimensionless time, $t u / L$ \\
\hline$N$ & number of $y$-steps & $\sigma$ & real part of $p$ \\
\hline$p$ & Laplace complex variable & $\tau$ & Fourier number, $D_{\mathrm{m}} L /\left(u a^{2}\right)$ \\
\hline
\end{tabular}

\section{THEORETICAL CONSIDERATIONS}

The numerical inversion of a given Laplace domain function according to the methods mentioned above requires the calculation of this function for a given set of values of the Laplace complex variable $(p)$. This set is determined by the method selected for performing the inverse Laplace transformation (e.g. $p_{k}=k$ where $k=1,2,3, \ldots$, for the method using expansion of the function in series of Chebyshov polynomials of the first kind; $p_{k}=2 k+1$ where $k=1,2,3, \ldots$, for the method employing Fourier sine series expansion) [2]. The set of function values calculated in this way are used further on according to the algorithm of the preselected numerical inversion method for obtaining the corresponding time-domain function.

If differential equations are to be solved by the approach mentioned above then as a preliminary step their solutions in the Laplace domain must be obtained. This is in fact the traditional application of the numerical inversion methods for the solution of linear partial or ordinary differential equations when analytical inverse transformation is either not possible or the numerical inverse transformation is much faster than the direct use of the corresponding time-domain funcion. The latter is usually the case when the analytical inverse transformation leads to functions containing infinite series or integrals which must be solved numerically for each time point $[4,5]$.

For more complicated partial differential equations (e.g. multi-dimensional or with coefficients which are not necessarily constants) the Laplace transformation does not lead to a readily solvable ordinary or partial differential cquation. Howcver, the transformed equation has one independent variable less than the original time-domain equation. The computation time necessary for its numerical integration is therefore much less than that for the original equation. For this reason even if the transformed equation is solved several times for different $p$ values to provide the necessary function values for the numerical inversion algorithm, the total computation procedure, including the numerical inversion itself, will still remain much faster than the conventional integration of the original equation. Another advantage of the combined technique is that the solution is in the form of a continuous function of time while conventional integration leads to a discrete function of time which is less convenient for further processing (e.g. differentiation) than the continuous function.

\section{APPLICATION TO MASS-TRANSFER IN SINGLE AND MULTI-STREAM LAMINAR PARALLEL-PLATE FLOW SYSTEMS}

The equations describing the transient mass-transfer in single or multi-stream laminar parallel-plate flow systems are parabolic partial differential equations of the second order usually with three independent variables (one temporal and two spatial) and with coefficients not all of which are constants. In the general case these equations cannot be solved analytically. $\Lambda$ numerical method usually used for the solution of similar problems is the implicit alternatingdirection finite-difference method (IADFDM) [3].

In the subsequent paragraphs the equations mentioned above will be solved by both the method proposed in the present paper and the IADFDM. A comparison will be made between the two methods both with respect to precision and consumption of computation time.

\section{Mass-transfer in laminar parallel plate flow}

The convective-diffusion equation describing the mass-transfer in laminar flow between two parallel plates in dimensionless quantities and variables has the following form [6]

$$
\frac{\partial C}{\partial \theta}=\tau \beta \frac{\partial^{2} C}{\partial X^{2}}+\tau \frac{\partial^{2} C}{\partial Y^{2}}-U(Y) \frac{\partial C}{\partial X}
$$


where $U(Y)=1.5\left(1-Y^{2}\right)$ is the dimensionless flow rate, $\tau=D_{\mathrm{m}} L / u a^{2}$ is the Fourier number, and $\beta=(a / L)^{2}$ is a geometric dimensionless group.

It was shown that for most practical cases the axial diffusion term [i.e. $\tau \beta\left(\partial^{2} C / \partial X^{2}\right)$ ] can be neglected [6]. Such a simplification is used in the present paper without limiting the generality of the conclusions made.

The initial and boundary conditions under which this equation will be solved correspond to the case of two impermeable walls and a solute step-function input at $X=0$

$$
\begin{aligned}
& C(0, X, Y)=0 \quad(X \geqslant 0) \\
& C(0,0, Y)=1 \quad(0 \geqslant 0) \\
& \left(\frac{\partial C}{\partial Y}\right)_{r=0}=\left(\frac{\partial C}{\partial Y}\right)_{r=1}=0 .
\end{aligned}
$$

By Laplace transformation equation (3) is reduced to a partial differential equation with two independent variables (equation (4))

$$
\tau \frac{\partial^{2} \bar{C}}{\partial Y^{2}}-U(Y) \frac{\partial \bar{C}}{\partial X}-p \bar{C}=0
$$

The boundary conditions of equation (4) are

$$
\begin{aligned}
\bar{C}(0, Y) & =1 / p \\
\left(\frac{\partial \bar{C}}{\partial Y}\right)_{Y=0} & =\left(\frac{\partial \bar{C}}{\partial Y}\right)_{Y=1}=0
\end{aligned}
$$

Equation (3) was solved by the IADFDM [3]. The corresponding implicit finite-difference equations are given in the Appendix. The mean concentration in the cross-section of the flow at $X=1$ was calculated for each time point. Equation (4) was solved by the implicit difference method [3] (see the Appendix) for $p_{k}=2 k+1$ where $k=0,1,2, \ldots, 19$. In a similar way the Laplace transform of the mean concentration in the cross-section of the flow at $X=1$ was determined for each $p_{k}$. The numerical inverse Laplace transformation was performed by the method employing expansion of the Laplace domain function into Fourier sine series [2]. It was found that the truncation crror at the 15 th term in the infinite series constituting the final inversion formula (equation (5)) was already negligible

$$
C(\theta)=\sum_{k=0}^{k=x_{k}} b_{k} \sin ((2 k+1) \arccos [\exp (-0)])
$$

where

$$
b_{k}=\frac{4^{k+1}}{\pi} \bar{C}\left(p_{k}\right)-\sum_{i=0}^{k-1} \frac{2 i+1}{2 k+1} \frac{(2 k+1) !}{(k+i+1) !(k-i) !} b_{i} .
$$

The concentration-time profiles at $X=1$ calculated by the two methods were virtually indistinguishable
Table 1. Comparison between the proposed method and the $\operatorname{IADFDM}(\tau=1.0)$

\begin{tabular}{lccc}
\hline & \multicolumn{3}{c}{ New } \\
& 0 & method & IADFDM \\
\hline & 0.75 & 0.044 & 0.046 \\
Concentration & 1.00 & 0.511 & 0.511 \\
at $X=1$ & 1.25 & 0.878 & 0.874 \\
& 1.50 & 0.984 & 0.983 \\
Computation time $[\mathrm{s}]$ & & 86 & 1740 \\
\hline
\end{tabular}

from each other which is illustrated in Table 1. The proposed method is much faster because of the following reason : according to the IADFDM [3] for the determination of the concentration at $X=1$ for each time point two sets of systems of algebraic equations must be solved. The former set consists of $N+1$ systems of $M$ difference equations which are implicit in the $X$-direction, where $N+1$ and $M+1$ are the numbers of grid points in the $Y$ - and $X$-direction, respectively. The latter set includes $M$ systems of $N+1$ difference equations implicit in the $Y$-direction. The transient part of the concentration-time curve at $X=1$ for Fourier numbers from 0.5 to 3.0 is located in the dimensionless time region from 0 to 2 [6]. By the method of trial and error it was found that this time interval should be subdivided in 200 steps $(K=200)$. In this case the two sets of systems of difference equations mentioned above must be solved 200 times. At the same time for the numerical integration of equation (4), provided that the network of spatial grid points is the same, only one set of $M$ systems of $N+1$ implicit difference equations should be solved. This fact shows that if the computation time necessary for solving the three sets of systems of equations mentioned above is the same, the solution of equation (4) requires approximately $2 K$ times less computation time than equation (3). Taking into consideration that equation (4) should be solved 16 times it can be concluded that for this particular example the proposed method should be 25 times faster than the IADFDM. The real computation times for equations (3) and (4) are presented in Table 1. They show that the proposed method is 20 times faster than the IADFDM. This slightly lower value can be explained by the fact that the set of $X$-implicit equations for equation (3) can be solved faster than the $Y$-implicit equations which are similar in structure with the implicit equations of equation (4) (see the Appendix). The computation time necessary for performing the inverse Laplace transformation once the necessary set of function values are calculated is $6 \mathrm{~s}$.

Mass-transfer in a co-current parallel plate laminar double stream flow system

This flow system, consisting of two co-current parallel plate laminar streams (i.e. donor and acceptor streams) separated by a permeable with respect to the solute wall with zero resistance, is described math- 
Table 2. Comparison between the proposed method and the IADFDM ( $\tau=1.0$ for both the donor and the acceptor streams)

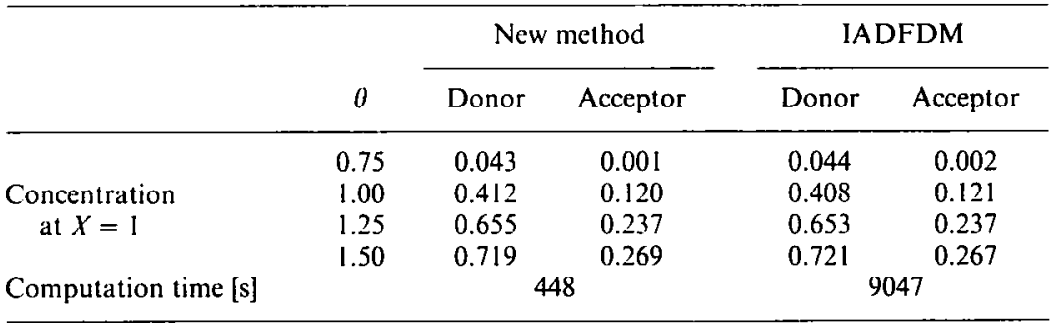

ematically by two equations similar to equation (3). They are coupled through joint boundary conditions and thus form a conjugated boundary value problem. A step-function concentration change in the donor stream at $X=0$ was assumed to take place at $\theta=0$. The details of the solution are reported elsewhere [7]. In the calculations it was assumed that both channels were of equal height and that the molecular diffusion coefficient of the solute in both streams had the same valuc.

Very good agreement was again observed between the proposed method and the IADFDM (Table 2) and the ratio of the corresponding computation times was almost the same as in the previous example, i.e. 20.2 .

\section{CONCLUSIONS}

A fast numerical method for solution of linear partial differential equations frequently encountered in mathematical physics and chemical engineering describing transient transfer phenomena was developed. It is based on transforming the equations in the Laplace domain where they are numerically solved and subsequent numerical inverse transformation of these solutions. The computation process can be spceded up by more than one order of magnitude in comparison with the numerical techniques for approximate solution of linear partial differential equations traditionally applied in such cases. In the present study the finite-difference methods were used for the solution of both the original and the Laplace transformed equations. It should be pointed out that any other appropriate method for approximate solution of linear partial differential equations can be used as well. This fact shows that any improvement in the numerical techniques for solution of linear partial differential equations could be utilized successfully for improving the method described in the present paper. As far as the numerical solution of transicnt multi-dimensional transport problems is time-consuming the proposed method also provides a substantial economy of computation time in absolute value, especially when multiple solving of the corresponding equations is required. This becomes very crucial for multi-stream systems. The examples in the present study illustrate how sub- stantially the computation time increases with changing the number of streams from one to two.

The examples considered in the present investigation are equations describing transient masstransfer in laminar flow systems. Because of the similarity between mass- and heat-transfer it can be concluded that the proposed method is suitable also for the solution of equations describing transient heattransfer in single and multi-stream flow systems.

\section{REFERENCES}

1. F. B. Hildebrand, Advanced Calculus for Applications. Prentice-Hall, Englewood Cliffs, New Jersey (1962).

2. S. D. Kolev and E. Pungor, Numerical solution of hydraulic models based on the axially-dispersed plug flow model by Laplace transforms, Anal. Chim. Acta 194, 6175 (1987)

3. B. Carnahan, H. A. Luther and J. O. Wilkes, Applied Numerical Methods. Wiley, New York (1969).

4. S. D. Kolev, K. Toth, E. Lindner and E. Pungor, Flowinjection approach for the determination of the dynamic response characteristics of ion-selective electrodes. Part 1. Theoretical considerations, Anal. Chim. Acta 234, 49-56 (1990).

5. S. D. Kolev, G. Nagy and E. Pungor, Mathematical modelling of single-line flow-injection analysis systems with single-layer enzyme electrode detection. Part 1. Development of the mathematical model, Anal. Chim. Acta 241, 43-53 (1990).

6. S. D. Kolev and W. E. van der Linden, Laminar dispersion in parallel plate sections of flow systems used in analytical chemistry and chemical engineering, Anal. Chim. Acta 247, 51-60 (1991).

7. S. D. Kolev and W. E. van der Linden, Analysis of transient laminar mass-transfer in a parallel-plate dialyser, Anal. Chim. Acta 257, 331-342 (1992).

\section{APPENDIX}

The $\theta, X$ and $Y$ regions of interest are divided into $K, M$, and $N$ subintervals, respectively, so that it can be written:

$$
\begin{array}{ll}
\theta=k \Delta \theta & (k=0,1,2, \ldots, K) \\
X=i \Delta X & (i=0,1,2, \ldots, M) \\
Y=j \Delta Y & (j=0,1,2, \ldots, N) .
\end{array}
$$

Implicit finite-difference equations of equation (3)

These equations form the following two sets of systems of algebraic equations:

set of $N+1$ systems $(j=0,1,2, \ldots, N)$ of $M X$-implicit equations $(i=0,1,2, \ldots, M-1)$ : 
Table AI. Coefficients of the finite-difference equations of equations (3) and (4)

\begin{tabular}{cccc}
\hline & 0 & $0<j<N$ & $N$ \\
\hline$D_{0}$ & $2 d_{1} C_{i, 1, k}$ & $d_{1}\left(C_{i, j-1, k}+C_{i, j+1, k}\right)$ & $2 d_{1} C_{i, N-1, k}$ \\
$D_{1}$ & 0 & $-d_{1}$ & $-2 d_{1}$ \\
$D_{2}$ & $-2 d_{1}$ & $-d_{1}$ & 0 \\
$D_{3}$ & 0 & 1 & 2 \\
$D_{4}$ & 2 & 1 & 0
\end{tabular}

$\left(1+d_{2}\right) C_{i, j l+0.5}-d_{2} C_{i-1 . j k+0.5}=D_{0}+\left(1-2 d_{1}\right) C_{i, j k} \quad(\mathrm{Al})$

where $d_{1}=\tau\left(\Delta 0 / 2(\Delta Y)^{2}\right) ; d_{2}=0.75\left[1-(j \Delta Y)^{2}\right](\Delta 0 / \Delta X)$ : and $D_{0}$ are defined in Table $\mathrm{Al}$ : set of $M(i=1,2, \ldots, M)$ systems of $N+I Y$-implicit equations $(j=0,1,2, \ldots, N)$ :

$D_{1} C_{t, j-1, k+1}+\left(1+2 d_{1}\right) C_{i, j k+1}$

$$
+D_{2} C_{i, j+1, k+1}=d_{2} C_{i-1, j, k+0.5}+\left(1-d_{2}\right) C_{i, j, k+0.5}
$$

where $D_{1}$ and $D_{2}$ are defined in Table Al.

Implicit fimite-difference equations of equation (4)

These equations constitute $M$ sets $(i=0,1,2, \ldots, M-1)$ of $N+1$ implicit algebraic equations $(j=0,1,2, \ldots, N)$ $D_{3} \bar{C}_{i+1, f_{1}}-\left(2+g_{1}\right) \bar{C}_{i+1, j}+D_{4} \bar{C}_{i+1, j+1}=\left(g_{2}-g_{1}\right) \bar{C}_{i, j}$

where $g_{1}=(1.5 / \tau)\left[1-(j \Delta Y)^{2}\right]\left((\Delta Y)^{2} /(\Delta X)\right) ; \quad g_{2}=(p / \tau)$ $(\Delta Y)^{2} ;$ and $D_{3}$ and $D_{4}$ are defined in Table Al. 